Relations industrielles

Industrial Relations

\title{
Organisation du travail dans l'entreprise, par Neeby D. Gardener, traduit et adopté par Michel de Tihef, Édition Gamma, Paris, 1966, 119 pages.
}

\section{Bernard Solasse}

Volume 24, numéro 1, 1969

URI : https://id.erudit.org/iderudit/028007ar

DOI : https://doi.org/10.7202/028007ar

Aller au sommaire du numéro

Éditeur(s)

Département des relations industrielles de l'Université Laval

ISSN

0034-379X (imprimé)

1703-8138 (numérique)

Découvrir la revue

Citer ce compte rendu

Solasse, B. (1969). Compte rendu de [Organisation du travail dans l'entreprise, par Neeby D. Gardener, traduit et adopté par Michel de Tihef, Édition Gamma, Paris, 1966, 119 pages.] Relations industrielles / Industrial Relations, 24(1),

225-226. https://doi.org/10.7202/028007ar

Tous droits réservés @ Département des relations industrielles de l'Université Laval, 1969
Ce document est protégé par la loi sur le droit d'auteur. L'utilisation des services d'Érudit (y compris la reproduction) est assujettie à sa politique d'utilisation que vous pouvez consulter en ligne.

https://apropos.erudit.org/fr/usagers/politique-dutilisation/ 


\section{Partie 8: Le système d'entreprise - le} système des valeurs

Notre système d'entreprise, ses buts et les moyens employés pour les atteindre, est-il en harmonie avec le système des valeurs que privilégie notre société démocratique et de bien-être ? Les administrateurs de ce système d'entreprise se nourrissent-ils au commun système de valeurs qui a pour objectifs la stabilité économique, l'investissement dans la production de biens dont le besoin est réel, la distribution équitable des richesses, la production la plus efficace possible des biens demandés, la contribution du système économique au développement de structures politiques et sociales saines ? $\mathrm{La}$ réponse à ces questions nécessiterait l'analyse et la détermination des structures de chacun de ces systèmes.

\section{Partie 9: La culture - la croissance}

L'activité productrice de l'entreprise s'inscrit dans un milieu plus vaste où ont cours différentes philosophies où sont posés une multiplicité de gestes quotidiens: ce milieu plus vaste c'est la culture. Celle-ci a un aspect dynamique dont l'éventuel impact sur la croissance de l'organisation fait l'objet des discussions de ce chapitre: Ce qu'est la culture, ce qu'est la croissance, ce qui résulte de leur interpénétration.

\section{Partie 10: Le monde - l'idéologie}

Le but ultime de l'administrateur étant le progrès humain à une échelle de plus en plus mondiale, nait la nécessité de s'interroger sur le rôle qu'il faut jouer dans la promotion d'une idéologie autour du monde. Encore ici sont préalablement définis les concepts employés.

\section{Hervé GAUTHIER}

Pour une doctrine de l'entreprise, par Philippe de Woot, Editions du Seuil, Paris, 1968, 285 pages.

L'auteur veut ajouter aux publications précédentes qui avaient ouvert le débat sur l'entreprise. Il rappelle d'abord le débat et les réformes proposées, de même que les diverses idéologies patronales et leurs répercussions.

Philippe de Woot remet, lui aussi, en question, l'entreprise. Il s'interroge à savoir «qu'est-ce que l'entreprise?» Pour cerner l'entreprise il fera appel à trois notions : l'entrepreneur, l'organisation, le pouvoir. Il tentera de les situer objectivement dans les situations de marchés européens et américains, en donnant les différences spécifiques de ces marchés.

L'auteur fait une étude scientifique de l'entreprise et essaie de voir comment l'entreprise peut répondre aux attentes de plusieurs groupes d'intérêts qui, présentement, contestent l'entreprise traditionnelle.

Enfin, il expose des éléments d'une doctrine de l'entreprise. Il définit l'acte d'entreprendre: «Mettre en oeuvre des ressources en vue de créer et de distribuer des biens et des services d'une manière profitable et cumulative, dans un milieu en évolution constante $\gg$. La fonction principale de l'entreprise sera la créativité.

Dans sa conclusion, l'auteur fait remarquer que l'entreprise doit être forte et évoluer profondément. Selon de Woot, la créativité, la participation et la concertation sont les trois dimensions d'une entreprise qui se veut capable d'entreprendre dans le monde de demain.

Ce volume a valu à l'auteur d'être le premier lauréat de la fondation Léon Bepaert.

\section{Pierre BRIEN}

\section{Organisation du travail dans l'entreprise,} par Neeby D. Gardner, traduit et adopté par Michel de Tihef, Edition Gamma, Paris, 1966, 119 pages.

Cet ouvrage est le second volume d'une série de cours dits « d'enseignement programmé ». Le but poursuivi dans ce second volume est d'accroître l'efficacité de l'entreprise en améliorant l'organisation du travail.

L'originalité du livre tient moins aux idées développées sur l'organisation du travail qu'à la pédagogie utilisée.

Toute idée avancée par l'auteur est aussitôt assortie de questions; et le lecteur est appelé à choisir parmi plusieurs réponses à cette question, celle qu'il estime la plus juste.

Dans un second temps l'auteur explique ce qu'est la bonne réponse et pour- 
quoi elle est la bonne et pour se faire utilise les réponses fausses que le lecteur aurait pu choisir.

Ce livre s'adresse surtout aux praticiens et c'est là son intérêt.

\section{Bernard SOLASSE}

La France et le management, par Roger Priouret, Denoël, Paris, 1968, 407 pages.

Après le Défi Américain de Servan Schreiber, Priouret expose les problèmes de son pays mais fait appel à des personnalités connues pour leurs succès administratifs afin de donner une image plus dynamique de la France.

Dans une première partie, Priouret rappelle la situation patronale française et le rôle économique du gouvernement comme une source du malaise actuel. De plus, il souligne qu'il ne faut pas engager des batailles perdues d'avance. Il faudrait aussi effectuer une mise sous tension rationnelle de l'ensemble des éléments qui composent une entreprise. Enfin, il rappelle qu'il conviendrait de réhabiliter la notion de profit.

La seconde partie repose sur de précieux témoignages qui reposent sur les sujets suivants: l'autofinancement, la participation aux profits, le recrutement et le traitement des cadres, la décentralisation de l'entreprise, la diversification des produits, et l'évolution générale des entreprises qui sont sous la gestion des interviewés.

\section{Pierre BRIEN}

\section{Unemployment, Money Wage Rates,} and Inflation, by George L. Perry; The M.I.T. Press, Boston, 1966, pp. $\mathrm{XV}, 143$.

Le livre de Perry utilise les modèles développés par Phillips et Lipsey pour analyser les relations salaire, emploi et prix aux Etats-Unis. Il s'agit d'une des études les plus importantes dans ce domaine, jamais entreprises aux EtatsUnis. L'auteur fait d'abord un rappel des principales études déjà faites sur les équations de salaire; puis il bâtit son propre modèle d'analyse de régression mul- tiple où en plus des traditionnelles variables, salaire, chômage et prix, il introduit le niveau et les variations de profits parmi les variables inclépendantes. Les observations portent sur des données trimestrielles d'après-guerre. De plus, comme l'auteur construit ses variables sur la base des variations annuelles ramenées en pourcentage, il suit que chaque observation retenue contient trois trimestres de l'observation précédente. S'il existe un certain degré de corrélation entre les trimestres et les variations de salaires, cette méthode a pour effet d'introduire de l'autocorrélation des erreurs résiduelles. L'auteur consacre plusieurs pages pour traiter de cette question.

Les périodes couvertes par l'étude du professeur Perry portent principalement sur l'après-guerre, néanmoins certaines de ses estimations remontent à 1920 . Les résultats qu'il obtient pour les trimestres compris entre 1948 et 1960 se ramènent à l'équation suivante pour l'ensemble de l'industrie de transformation aux EtatsUnis :

$$
\begin{aligned}
& \mathrm{W}_{\mathrm{t}}=-4.313+0.357 \mathrm{C}_{\mathrm{t}-1}+14.711 \mathrm{U}_{\mathrm{t}-1} \\
& \text { (0.054) } \\
& +0.424 R_{t-1}+0.796 \Delta R \text {. } \\
& \text { (0.068) } \\
& \mathbf{R}^{2}=.870
\end{aligned}
$$

où $\mathrm{W}_{\mathrm{t}}$ désigne les variations annuelles de salaire, $C$ les variations annuelles de l'indice des prix au consornmateur, et U le taux de chômage, R, le taux de profit.

A partir de cette équation, le professeur Perry construit plusieurs jeux de "trade-off". Par exemple, substituant dans l'équation des salaires la relation $\mathrm{C}=\mathrm{W}-\mathrm{P}$ où $\mathrm{P}$ est le taux de croissance de la productivité, il obtient les taux de chômage correspondant à certains taux donnés de productivité et de chômage ainsi qu'aux variations de salaires. En général les niveaux de chômage qu'il obtient en solutionnant les relations de "trade-off" sont relativement élevés. Ainsi avec un taux annuel de la croissance de la productivité de $3 \%$, la stabilité des prix est associée à un taux de chômage de $6 \%$. Dans ses analyses de "trade-off", Perry fait l'hypothèse que l'élasticité de substitution entre le travail et le capi- 